\title{
Unu idrònimu sardu
}

\author{
Mauru Maxia \\ Chircadore abilitadu \\ mauromaxia@libero.it
}

Resumu: Subra a sas orìgines de su topònimu Palau sos pàrreres de sos linguistas non sun cuncordes ma tottus creen chi siat una paraula foristera. Calicunu pensat chi siat essida a pizu a sos tempos de su domìniu catalanu-ispagnolu mentres àtteros sun cumbintos chi siat unu prèstidu cadalanu ebbia e bi est finas chie creet chi si trattet de unu ligurismu. S'artìculu mustrat chi s'esàmene de sa toponimia istòrica, màssimu de sos idrònimos, nos indittat intamen chi si trattat de unu topònimu chi s'est formadu comente isviluppu derettu de una paraula latina.

Paraulas crae: pàrreres; toponimia istòrica; idrònimos; isviluppu locale.

Abstract: On the origins of the name Palau scholars have different opinions but everyone thinks it's a foreign word. Someone thinks it's a thalassonim formed during the Catalan-Spanish period while others are convinced that it is a Catalan word and there are also those who believe that it is a Ligurian term. The article shows that the examination of the historical place names, and in particular of the hydronyms, indicates conversely that the name was formed as a direct local development of a Latin word.

Keywords: opinions; historical place names; hydronims; local development. 



\section{S'idrònimu pèlagu}

In mesu a sas paraulas chi che sun essende foras dae s'usu, ma chi su significu issoro est galu cumpresu dae sos chi faeddan in sardu, jughet unu postu de interessu pèlagu 'pischina, poju'. Sas variantes pèlau, pèalu, pelàu, palàu s'agattan pro su prus in forma de topònimos ispartighinados in paritzos puntos de s'Ìsula e de pretzisu:

1. Monte Pèlau o Pèalu de Siligo, Thiesi, Bessude (Pittau, 1997: 157);

2. Pèlau e Monte Pèlau de Borutta (ATS, inèditu);

3. Pèlau e Domo de Pèlau de Cossoine (Tetti, 2001: II, 403; Pittau, 2011: 187) chi in su situ web Sardegna GeoPortale est iscrittu cun sa forma faddida Domo de Palau;

4. Flùmini Pelàu, Pelàu, Pelàu Mannu e Pelaèddu de Cardedu, Gairo e Jerzu (ATS, 2006: 82);

5. Monti Palàu de Silìcua (presentzia de padules a pes de su monte);

6. Pelàia de Cossoine (Tetti, 2001: II, 403 "logu de pischinas").

In sa variante Pèalu de su n. 1 si osservat una metàtesi comente in su tuscanu pègalo (Malagoli, 1939: 283).

Pro tottu custos sitos sos cuntestos geomòrficos nos assiguran una presentzia de s'abba comente pischinas naturales, pojos, padules e benales. In particulare su Flùmini Pelàu, chi est unu de sos printzipales de s'Ozastra, innanti de isfogare in mare format paritzos pojos e una pischina manna dae ue paret chi nde leet su nòmene.

Sos datos e sos puntos de cussos topònimos si poden bìdere in su situ web Sardegna GeoPortale de sa Regione Autònoma de Sardigna (http://webgis.regione.sardegna.it/ricercatoponimi/). In custa serie toponìmica est capatze chi b'intret finas Serra Pelài de Elini.

Sa paraula pèlagu est documentada dae su XII sèculu in sas printzipales fontes iscrittas: Pelagu (CSNT: 108; 288; 302; 303; Stat.Sass.: I, 139, 46v; CDS: I, sec. XII, doc. 25, 198/1: Pelai); pelau de ponte de Turres (CSG: 10, 11. 22-23).

Dino Manca, curadore de su glossariu e de sos ìndices de su Condaghe de Santu Gaine, interpretat sa paraula pelau cun su significu de 'mare' (CSG: 72). Ma isse non tenet in contu chi in cussu passu de su 
documentu custu idrònimu est ligadu a istrintu cun sa paraula ponte (de Turres). Paret ladinu, tando, chi si trattet de sa matessi pischina manna chi s'agattat finas oe in currispondentzia de su ponte antigu (naradu Ponte Romanu) de su Riu Mannu de Portu Turres.

Max Leopold Wagner creiat chi su lessema in chistione esseret foras de usu (Wagner 1962: 242 s.v. pèlagu). Custa paraula, intamen, s'est mantesa in Ulìna finas cun s'antiga cunsonante velare sonora /-g-/ (Pittau, 2014: 619).

Custa paraula est galu in usu finas cun sa variante logudoresa palàu 'pantanu' (Berchidda) e in su modu de nàrrere "esser tottu a unu palàu" 'esser tottu infustu, illudrinadu, impantanadu' (Casu, 2002: 1021).

In sos ditzionarios sa forma pèlau est assentada cun su significu de 'padule, pantanu, terrinu abbosu' (Pittau, 1997: 157 s.v. Pelau), 'lagu minore (finas temporaneu)' (Pittau, 2014: 619).

Sa variante pelàu est rezistrada comente 'poju de abba in sos rios a zisa de istaniu' (Puddu, 2000: 1295). Pedru Casu, semper pro pèlau, rezistraiat finas su significu de 'mare' ma si trattat de unu pàrrere iscaminadu dae unu passu male cumpresu de su Condaghe de Santu Gaine de Turres ue si cunfunden sas paraulas ponte e monte (Casu, 2002: 1047 s.v. pèlau: "candho haet mancare abba in su pelau de monte de Turres"). Forsi si devet a una lèggida de custu autore su matessi intèrpretu de Dino Manca.

Sa variante palàu s'est mantesa finas in sos insediamentos rùsticos (istatzos) e in calchi burgada de sa Gaddura cun su significu de 'abbadorzu postu a tesu'. Lenardu Gana rezistrat sa boghe palau comente 'serbatoiu de abba; puttu naturale; bacinu naturale de paga istèrrida' offerende finas una interpretada de su significu de su topònimu gadduresu Palau (Gana, 1970: 421 s.v. Palau). In sa matessi linia est Salvadore Brandanu chi lu definit 'puttu naturale, bacinu, fossu, serbatoiu de abba' (Brandanu, 2004: 372 s.v. palàu). Arriscosa paret intamen sa definidura 'palaia de inibberos' chi proponet Andria Usai (Usai, 1977: 181) pro su fattu chi in sos chi faeddan su gadduresu non s'agattat peruna cunfirma de cussu significu. Sa matessi definidura est repitida in s'imprenta de su lèssicu de Usai chi nd'at torradu a fàghere Pascale Ciboddo (Ciboddo, 2003: 253).

Virgilio Tetti, cun s'iscrùpulu chi lu distinghiat, non mancheit de assentare chi "in Santu Tiadoru e atterue in Gaddura [...] lu palàu è lu fossu chi si faci in campagna, a longu, palchi li besti aggini l'ea in l' $i$ - 
stiu" "su palau est su fossu chi si faghet in campagna, a tesu, pro chi sas bestias appan s'abba in s'istiu' (Tetti, 2001: II, 403 s.v. Pelau).

Finas duos topònimos de s'Anglona dian pòdere èssere atterettantas disformaduras de sa matessi base. Su primu est s'idrònimu Puligosa (Maxia, 1994: 349) chi dessignat una boltada manna formada dae su Riu Coghinas peri sa làccana chi partit su territoriu de Bortigiadas dae cussu de Pèrfugas. Bi nd'at chi creen chi custu nòmene geogràficu siat formadu dae sa paraula pùliga e pro cussu naran chi siat unu 'logu de pùligas'. A nàrrere sa veridade, custos puzones non bi mancan in su cursu de su Coghinas, ma est sa desinentzia -òsa chi faghet bènnere su suspettu chi si trattet de una forma paretimològica pro pelagosa. Tambene, in sa formatzione de sos nòmenes geogràficos a sos zoònimos si lis assotziat de preferentzia su suffissu -àrgiu, -àrzu; pro cussu, si su topònimu nde benzeret dae pùliga, si diat isettare una forma comente *puligarza sigundu modellos che baccarza (bacca + -àrza), corvarza $($ corvu $+-a ̀ r z a)$, crabargia (craba +-àrgia) e sìmiles.

Su sigundu topònimu est Santu Pedru Puligosu de Pèrfugas (Maxia, 1994: 391), ue s'aggettivu puligosu si creet chi nde benzat dae pùlighe. Ma finas in custu casu est capatze chi siat intervènnida una banalizadura pro unu accostamentu a pùlighe intamen de una forma prus antiga chi podiat currispòndere a *pelagosu. Non paret de badas chi a pagu bettu dae sa ruina de cussa cresiedda intitulada a Santu Pedru s'agattet una pischina manna formada dae su Riu Anzos e chi est prus connotta comente Pischina de Piraùla (coord. 4048'24.38"N 8 53'01.09"E). Custu significu de pischina naturale o de poju s'agattat ebbia in tuscanu dae s'incumintzu de su XIV sèculu cando formaiat finas topònimos, p.es. in sos Istatutos Senesos (ed. Gangalandi, 1309-10: dist. 3, cap. 156, vol. 2, p. 72.19):

\section{Etimologia}

S'ètimu de s'appellativu in chistione, chi s'agattat sempre cun significos de idrònimu, si sebestat in su lat. pelăgu(m) (REW 6369; Pittau, 2014: 619) chi benit dae su gregu pélagos (Ernout-Meillet, 1951: 874). In sas variantes chi presentan s'ispostamentu de s'accentu subra a sa sillaba chi sighit su fenòmenu paret chi si siat avverguadu a pustis de s'iscumpàrfida de sa velare /-g-/, regulare in cuntestu intervocàlicu, chi est documentada dae su XVI sèculu cun sa grafia pelau in su Condaghe 
de Santu Gaine. Est de nàrrere finas chi non mancan casos affattentes a custu de pèlagu > pèlau chi presentan ebbia s'iscumpàrfida de sa velare sonora paris cun s'ispostamentu de s'accentu subra a sa sillaba chi sighit. Custu fattu si podet bìdere in sa variante isparàu pro ispàrau dae ispàragu (lat. asparagu(m) o finas in sa variante cannàu pro cànnau dae cànnagu, cànnabu (lat. cannabu(m). In su Cabu de Josso, ue si impittat sa variante palàu comente in Gaddura, in cunditziones anàlogas su passazu $e>a$ lu bidimus finas in su topònimu Santu Sparàu a costazu de Santu Speràu (ant. Santu Speradu).

$\mathrm{Su}$ matessi fenòmenu si podet bìdere in manera finas prus ladina in sa paraula thàlau, chi sos istudiosos apponen a su sustratu preromanu. Custa paraula, chi comente palàu presentat sa sillaba tònica pretzèdida dae /-1-/, tenet sa variante thalàu cun su matessi ispostamentu de accentu subra a sa sìllaba chi sighit. Custas chi amus bidu, màssima custa ùltima, sun formas chi dian pòdere àere àppidu una funtzione de modellu pro su passazu pelàu > palàu.

In sa variante palàu su passazu $e>a$ in protonia espressat, siat in sardu (Wagner-Paulis, 1984: 46 segg.) siat in gadduresu, unu fattu regulare dae nantis a cunsonantes lìcuidas e nasales (cfr. gadduresu cunsal-

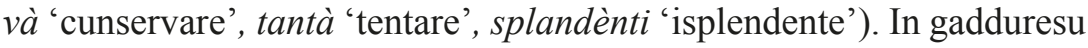
pustis su fenòmenu est regulare màssimu dae nantis a /r/ finas cando passat a /1/ pro sa tendentzia de custa variedade a su lambdacismu. Ma su fenòmenu s'avverguat finas in àtteros cuntestos comente in sos casos de accìsu < isp. echizo, attugnu < isp. otoño e àtteros (Maxia, 2012 : 91-92). In custu casu est finas de osservare chi su gadduresu in protonia preferit su trattamentu $e>i$; duncas dae pèlagu si diat dèvere isettare una forma comente pilau. E tambene su cossu lu jughet s'isviluppu pilagu (Falcucci, 1915: 275). Ma custa forma no esistit in su gadduresu ca custa limba, comente semus bidende, pro su fenòmenu in chistione non s'accordat cun su cossu ma cun su sardu. In su passazu el $>a l$, posca, si bi diat pòdere finas reconnòschere un'influssu de sa paraula palu comente mustrat s'intèrpretu de Andria Usai e finas su pàrrere de Massimo Pittau (mira prus innanti).

De importu est sa forma Palài (Alà, Anela, Bolotana, Bottidda, Mores, Ollolai, Sèneghe) e in particulare Riu Palài (Atzara, Bonorva, Boroneddu, Ghilarza) (Paulis, 1987: 443) pro su fattu chi mustrat de èssere un'idrònimu gasi comente sa paraula pèlau e sas variantes chi dae issa nde sun bènnidas. Benevenuto Terracini, dae parte sua, firmèndesi a 
s'apparentzia de custa forma, assenteit custu geònimu inpare a sos derivados de pala 'costa di monte' (Terracini, 1926 : 131).

De interessu paren finas sos idrònimos Funtana Palàxi e Laccu Palàxi (Biddesartu/Villasalto), Serra Palàxi (Santu Basili de Monte/ San Basilio) ue a sa raiga pal-si ligat su suffissu -àxi. Dae parte sua, su talassònimu Balài (Portu Turres) est un'isviluppu sighidu a s'iscumpàrfida regulare de /-g-/ intervocàlica (Wagner-Paulis, 1984: 135-6) de sa forma antiga Balagai (Spanu, 2000: 121) chi tenet una istruttura cunsonàntica simile a cussa de lat. pelăgu(m).

Custas ùltimas formas, chi dian pàrrere preromanas, nos indittan chi in Sardigna innantis chi lomperet sa boghe lat. pelăgu $(\mathrm{m})$ bi deviat cùrrere una raiga idronìmica "mediterranea" *PAL- (Blasco Ferrer, 2010: 127) de cunfrontare, subra ambos pianos fonomorfològicu e semànticu, cun su lat. palus de orìzine indeuropea (Ernout-Meillet, 1951: 847) chi diat pòdere dare contu de su trattamentu pal- pro pel- in sa variante palàu. Ultres de cussu, sos puntos geogràficos de sas variantes e de sos topònimos chi amus bidu cunsizan de leare in cunsideru chi, mancari sa forma lat. pelăgu(m) appat prevàlidu, s'impreu de s'antiga forma preromana siat sighidu in sos puntos ue s'est mantesa sa variante palàu (Gaddura, Berchidda, Ozastra, Siliqua) irrughèndesi cun sos isviluppos romanzos.

\section{Su topònimu Palàu}

S'arresonu chi pertoccat a pèlagu e a sas variantes suas interessat finas sa chistione de sa nàschida de su topònimu Palàu. Subra a custu topònimu est essidu dae pagu unu volumeddu de su palaesu Emilio Aresu chi cabet un'artìculu intituladu Storia di una Sorgente (Aresu, 2014b: 131-140) ue s'autore chircat de agattare s'ètimu. Pustis de una rassigna de sos iscrittos e de sas cartas geogràficas de sos ùltimos duos sèculos, ue cumparet su topònimu cun variantes gràficas divessas, isse presentat unu suntu de sas propostas etimològicas avantzadas dae cussos chi si sun interessados, dae paritzos livellos, de custa chistione. In sighida signalat su fattu chi finas dae printzipios s'afficcu issoro s'est appuntadu, pro su prus, a sa variante ligure $U$ Parà (Bonifatu) e U Paràu (Sa Madalena) e chi custu at cunditzionadu s'orientamentu de paritzos ispertos.

Aresu si proponet, dae un'ala, de dimustrare chi su nòmene geogràficu Palàu representet unu idrònimu e, dae s'attera, chi sa nàschida 
sua nde benzat dae sa limba grega chi, sigundu isse, diat èssere istada faeddada dae sos antigos Cossos chi istaian in Gaddura innantis de sa conchista romana. Pro sa prima chistione si podet èssere de accordu cun s'autore ca portat datos avverguàbiles e de profettu pro s'arresonu subra a su topònimu. A cunfirma de custu, paret de importu sa prima documentatzione cartogràfica de su situ, de su 1812, ue figuran s'unu accurtzu a s'àtteru su topònimu Il Parao e s'iscritta Acqua (Piloni, 1974: 217). Finas una carta inglesa imprentada una bindighina de annos in fattu (1827) presentat inpare Parau e Water (Piloni, 1974: 217). Ambas fontes cartogràficas signalaian, duncas, chi su logu naradu Parao o Parau fut nòdidu pro sa presentzia de abba. Abba chi, comente si podet cumprèndere, fut abba de bìere, est a nàrrere unu elementu de importantzia manna pro sa zente de mare chi aiat bisonzu meda de connòschere sos sitos ue beniat bene a fàghere provvista de abba dulche. Duncas si podet crèere chi sas grafias acqua e water de cussas cartas esseren calcos semànticos de una forma locale chi marcaiat sa presentzia de abba dulche. Cussa forma currispondet guasi a seguru a su situ, como urbanizadu, naradu Lu Palàu chi pustis est devènnidu su nòmene de su populadu. In currispondentzia de cussu situ unu tempus essiat unu càntaru chi posca est istadu in parte covaccadu dae sa linia ferrada (com. pers. de Mario Scampuddu). In un'àtteru situ naradu Lu Palau Vecchju (Aresu, 2014b: 132) unu càntaru prus bundante at alimentadu pro deghinas de annos su sabunadorzu pùbblicu a pagu bettu dae su portu turisticu (com. pers. de Mario Scampuddu). Ambos sitos sun ammentados in sa toponimia urbana cun sas formas italianizadas Palau Vecchio e Fonte Vecchia. A dolu mannu, sas mudantzias casionadas dae su isviluppu ediliziu como non permittin de avverguare si in printzipiu sa paraula palàu esseret referida a unu de cussos càntaros o a calchi fossu o poju chi sas abbas issoro podian formare in àtteros tempos. Su chi paret siguru, intamen, est chi sa paraula in chistione indittaiat una presentzia de abba bona a bìere e no unu puntu de sa costa impreadu comente portu o iscalu de imbarcatziones.

Dae su puntu de vista gràficu est de annotare chi in mesu a sas attestatziones litterarias e cartogràficas de su topònimu dae sa finida de su Settighentos a sos incomintzos de s'Ottighentos (Aresu, 2014b: 132) sun prus frecuentes sas variantes de tipu lìgure (Parào, Paràu) in cunfrontu a cussa autòctona (Palàu) chi in finis est intrada in s'usu uffitziale. Comente s'at a bìdere in sighida, sas formas lìgures, mancari 
appan unu significu divessu dae sa forma locale, sende guasi omògrafas an àppidu unu pesu mannu in sas propostas e in sos orientamentos etimològicos de unos autores chi andamus a bìdere.

In cantu a sa proposta etimològica, intamen, Aresu non lompet a conclusiones cumpartibiles ca isse ligat tottu s'arresonamentu a una tesi de fundu chi aiat espostu finas pagu tempus innantis (Aresu, 2014a: 165-170). Est una tesi, custa, chi previdet chi su topònimu Palàu nde benzat dae su grecu dòricu pálagos ma sena fagher contu subra a elementos cuncretos. Sos datos linguìsticos e toponìmicos ispartos in tottu su territoriu de Sardigna e chi amus bistu innanti mustran cale siat abberu sa situatzione dae su puntu de vista geolinguisticu e cantu siat dèbile cussa proposta. Sa chistione de sa limba faeddada dae sos antigos Cossos de Gaddura, chi Aresu e atteros militantes presumen chi esseret sìmile a su gregu sicilianu, est meda cumplicada e tenet bisonzu de unos iscrarimentos de fundu chi an a formare s'argumentu de unu cuntributu ispetzìficu chi est in sos dessignos de su suttiscrittu.

Beru est chi perunu de sos cummentadores, e finas sos ispertos chi si sun interessados de s'argumentu, s'est cunformadu a una metòdica de indagu chi, màssimu in toponomàstica, cheret sa cherta e s'esàmene de donzi datu o notissia chi tenzat utilidade pro s'incuadramentu de sa chistione dae unu puntu de vista linguìsticu. Sa fase ispetzialìstica, est a nàrrere s'esàmene de sos datos linguìsticos, non podet fàghere $\mathrm{a}$ mancu de cussa fase propedèutica. In toponomàstica custa metòdica est una conditio sine qua non si non si cheret arriscare de rùere in errores de intèrpretu. Donzi topònimu, gasi comente donzi paraula, tenet una propia istoria sua e, si sa linguìstica est un'iscentzia cumplicada, sa toponomàstica lu est finas de prus. Unu linguista chi s'accostet a s'etimologia sena avverguare unos datos netzessarios (documentarios, archivìsticos, istòricos, traditzionales, geomòrficos ecc.) curret sos matessi arriscos de cussos chi creen de pòdere ispricare orìzines e significos de sos nòmenes geogràficos cunfidende ebbia subra a sas formas gràficas. Medas boltas, e prus meda de cantu si pottat crèere, sas grafias sun s'esitu de banalizaduras, paretimologias, ipercorretziones e trobojos de paritzas zenias. Ma, mentres sas propostas de sos profanos sun de paga dura, cussas de sos ispertos cando sun male fundadas finin pro cunditzionare sa dibatta e custu custringhet sos àtteros istudiados a las iscumproare e finas a las respìnghere. $\mathrm{Si}$, posca, un'autore est nòdidu e reconnottu s'arriscu est chi sas propostas suas sian atzettadas e cunva- 
lidadas pro mediu de sa propia autoridade sua. In casos comente custu su dannu est finas prus mannu e lu dimustran medas etimologias de su canònigu Juanne Ispanu chi, mancari no esseren fruttu de datos reales, sighin a iscaminare e cunfündere sas ideas in fattu de toponomàstica sarda. Unu esempru pro tottus podet èssere cussu de un'àtteru idrònimu famadu, La Billèllera de Sosso, ue sighende a Ispanu finas un'ispertu abbistu comente Max Leopold Wagner s'est lassadu trazare in un'etimologia sena perunu fundamentu (Maxia, 2004: 39-52).

Dae s'àttera banda, si devet reconnòschere chi sos iscumproos e finas sos errores sun elementos netzessarios pro s'avantzamentu de sa connoschentzia, ca istìmulan e azian àtteros istudiados a chircare proas e argumentos noos chi permittan de s'accurtziare de prus a sa veridade. Ultres de cussu, cando s'orìzine de unas paraulas no est connotta (p.es. cun lessemas de sustratu), sas difficultades chi poden agattare sos istudiados sun finas prus mannas.

Custa premissa non paret sena utilidade ca finas un'istudiosu de cabbale comente Emidio De Felice (su primu a s'èssere interessadu de su topònimu Palau) accostèndesi a su significu de custu topònimu at offridu una proa chi non cumbinchet. Isse, tenzende contu ebbia de sa variante gràfica bonifatzina, chi presentat su rotacismu regulare de /-1-/ intervocàlica, proponzeit chi custu topònimu nde benzeret dae su cat.-isp. parar 'frimmàresi, arrèere', 'bardiàresi' cun referimentu particulare a sos deverbales paratge e paraje 'abbarru pro navios'. Sigundu isse custas duas paraulas si dian èssere cunformadas a sa fonètica e a su lèssicu de su logudoresu e de su gadduresu (De Felice, 1964: 97) ma sena nàrrere comente custu fattu diat èssere sutzessu. Cumparit cun evidentzia chi De Felice s'est firmadu a sas grafias ebbia de su topònimu e a su fattu chi cussu situ, agattèndesi peri sa costa, in sighida siat istadu impreadu comente nòmene de unu portu. Dae s'arresonamentu de De Felice essit a pizu chi su topònimu Palàu diat èssere unu talassònimu a sa matessi manera de medas nòmenes geogràficos postos in sa linia de costa e formados dae prestidos lessicales leados dae limbas foristeras. In veridade, si trattat de unu idrònimu chi pro cumbinassione ebbia s'agattat a pagu bettu dae sa ribba de su mare, comente at dimustradu Aresu chi connoschet a minudu su logu (Aresu, 2014b: 139).

Chi su topònimu Palàu siat unu idrònimu est proadu - ultres che dae sa currispondentzia formale cun topònimos sardos anàlogos ligados a sa presentzia de s'abba - dae s'esistentzia de unu topònimu idènticu, na- 
radu Fonti Lu Palau (http://www.montelimbara.it/? page_id=248), chi s'agattat in sos pendentiles de su Monte Limbara (Naitana, 2005: 3136). Custu topònimu, chi pertoccat a unu cantareddu de abba, pro su fattu chi s'agattat a 934 metros subra su livellu de su mare nde bogat calesisiat duda subra a sa possibilidade chi sa paraula palàu pottat àere unu rapportu semànticu cun sa presentzia de su mare.

In s'etimologia de su topònimu in chistione De Felice est ruttu in una faddina chi sos etimologistas poden cummittere sincasu chi non iscumproen cun contivizu mannu tottu sos elementos. In custu casu, dae su puntu de vista fonèticu isse at invèrtidu sos tèrmines de sa chistione interpretende sa forma Palàu comente unu adattamentu de su bonifatzinu Parà $(u)$ mentres sos datos e su chistionu dimustran chi sa forma bonifatzina diat poder èssere unu assebestu de cussa sarda.

Posca de una vintina de annos Eduardo Blasco Ferrer, forsis pensende chi Palau esseret unu toponimu formadu dae unu prèstidu cadalanu, indicheit su cat. palau 'palattu' a sa base de su topònimu, ma sena s'assigurare si in cussu logu in printzipios bi aeret àppidu unu palattu dae ue su topònimu nde aeret pòttidu essire a pizu in su tempus chi in Sardigna s'impreaiat su cadalanu (sèculos XIV-XVIII).

In sighida, gratzias a s'autoridade de De Felice, Massimo Pittau atzetteit cussa proposta annanghende "probabilmente con la intrusione del vocabolo palu 'palo', quello che serve per legare le imbarcazioni" (Pittau, 1997: s.v. Palau).

Pagos annos a como Fiorenzo Toso, indittende s'ètimu lat. palatu, est torradu a su cuntzettu de "palu" in s'ipòtesi de unu impreu antigu de su situ de Palau comente portu, duncas pensende finas isse, in manera anàloga a cussa de De Felice, a su topònimu Palàu comente una variante de su bonifatzinu $U$ Parà chi reflessat una de sas denominatziones lìgures pro 'scalo' (Toso, 2008: 33). In cussu arresonu jughet importu un'àtteru idrònimu palaesu, La Sciumara < lat. flumaria, chi benit abberu dae sa Liguria ue su lat. flumen dat sciûmme e est fàtzile a agattare formas sìmiles a cussa palaesa, p.es. sciûmmaea 'fiumara' (Gismondi, 1955: 333) e 'rivolo, rigagno, fossato' (Casaccia, 1851: 501), mancari medas boltas in sa toponimia uffitziale sian italianizadas cun sa forma Fiumara (Fiumara de Genua, Fiumara de Taggia).

Finas su suttiscrittu in printzipiu aiat leadu pro bona custa proposta de Toso intro de unu cuntestu geogràficu e istòricu chi bidet su situ de Palau ligadu a istrintu a s'influssu econòmicu e culturale ispartu pro 
sèculos dae sa colonia genuesa de Bonifatu (Maxia, 2011: 242; Maxia, 2012: 159, n. 2). E àtteros linguistas in cantu a La Sciumara (chi currispondet a sa foghe de su riu Liscia) bi an bistu unu neologismu ligadu a su turismu (Paulis, 2006: 20 "La Sciumara ossia la spiaggia dove avviene la "schiumata"').

Duncas, sos linguistas e lessicògrafos chi si sun accostados a sa chistione an postu afficcu a cuntzettos comente 'parare', 'palu' e 'palattu'. Ma niune at attuadu a avverguare si in su restu de s'Ìsula esisteren àtteros topònimos cun sa matessi forma o variantes chi aeren pòttidu àere su matessi intinu.

Virgilio Tetti intamen, pustis de àere cunsideradu sos sitos e sos cuntestos geomòrficos de sos topònimos chi li parian formados dae su lat. pelăgu(m), at assotziadu sa forma gadduresa palàu a sos isviluppos pèlau e pelàu. "Forse il porto di Palau, se non è voce catalana, deve il suo nome a una risorsa idrica" (Tetti, 2001: 403-404), est su cummentu de custu autore chi est su primu a àere avantzadu custa ipòtesi. Pro lòmpere a custa concluida isse tenzeit contu siat de su significu cumpartidu dae sas formas pèlau, pelàu e palàu e siat de su fattu chi in tottu sas situatziones avverguadas dae isse si cunfirmaiat sa presentzia de s'abba. Si devet a Tetti, tando, su primu cumprou de sa chistione dae unu puntu de vista pluridisciplinare e non solettantu linguìsticu.

Dionigi Panedda - un'àtteru istudiadu attentu meda - dae parte sua at rezistradu su topònimu Li Palài 'sas padules' pro Santa Justa (Loiri-Portu S. Paulu) e l'at ispianadu comente plurale de sa paraula gadduresa palàu 'palude, istagnu' (Panedda, 1991: 422, n. 1448).

Dae sas formas chi s'agattan in sa toponimia de paritzos comunes de s'İsula paret ladinu chi sa forma palàu e su topònimu idènticu chi s'agattat in Gaddura (Palau, Monte Limbara, Loiri-Portu S. Paulu) sun isviluppos regulares de su lat. pelăgu(m) sigundu sa trafila pèlagu > pèlau $>$ pelàu $>$ palàu. Duncas, si custu arresonu est bene fundadu, si diat trattare de unu de sos medas sardismos lessicales (prus de 3.500) de su gadduresu (Maxia, 2015) chi in custu casu faghet de base a unu topònimu chi, pro motivos istòricos, como est connottu finas foras de Sardigna comente portu e localidade turìstica. Chi si trattet de una paraula sarda no est unu casu particulare sende asseguradu chi finas dae sos primos sèculos de su sigundu millenniu sa Gaddura presentat topònimos meda chi documentan una preesistentzia de su sardu logudoresu 
(Maxia, 2012: 29-32; carta 43) chi in parte si osservat oe ettottu peri sas ìsulas linguìsticas de Luras, Terranoa (Olbia) e Fìgari (Golfo Aranci).

\section{Conclusiones}

Cun remiru a su lessema pèlagu, dae s'esàmene de sas grafias documentadas e dae s'anàlisi de sas variantes pèlau, pèalu, pelàu, palàu dae su puntu de vista fono-morfològicu e semànticu, ultres de sos cumproos geomòrficos, cumparet chi si trattat de formas chi sighin su lat. pelăg$u(m)$. A su variare de sas formas, difattis, non currispondet unu variare de sos significos ca sun anàlogos in tottu sa Sardigna dae sas alas de josso (Sulcis, Sarcidanu, Ozastra) finas a su Logudoro (Berchidda) e a sas estremidades de susu (Gaddura, Palau). Significos chi a minudu currisponden a 'poju de abba, pischina naturale, depòsitu (naturale o isfossadu), ludrau, lagu minore (finas de paga dura), pantanu, terrinu pantanosu, fossu pro dare a bìere a su bestiàmene'. Ultres de custos significos chi benin dae pelăgu(m), in una parte de sa Gaddura si osservat unu ispostamentu semànticu in su sensu de 'càntaru, funtana' chi est impreadu a costazu de sos àtteros significos chi sun semper in usu.

Pro su chi pertoccat a su topònimu Palàu, unos malintesos nàschidos dae grafias ligurizantes an discaminadu a sos istudiados in cunfrontu a àtteros incuadramentos chi bi podian èssere. Su cunfrontu cun àtteras formas e toponìmos presentes in su territoriu de s'İsula mustran abberu chi si trattat de un'idrònimu, passadu a topònimu, chi appartenet a su fundu latinu de sa limba sarda.

\section{Abbreviaduras}

$\begin{array}{llll}\text { ant. } & \text { antigu } & l l . & \text { lineas } \\ \text { cap. } & \text { capitulu } & n . & \text { nota } \\ \text { cat. } & \text { catalanu } & p . & \text { pàzina } \\ \text { cfr. } & \text { cunfronta } & \text { p.es. } & \text { pro esempru } \\ \text { com.pers. } & \text { comunicatzione personale } & \text { s.v. } & \text { sub voce } \\ \text { doc. } & \text { documentu } & \text { sec. } & \text { sèculu } \\ \text { ed. } & \text { editzione } & s e g g . & \text { sighentes } \\ \text { isp. } & \text { ispagnolu } & v & \text { revessu de su fogliu } \\ \text { lat. } & \text { latinu } & v o l . & \text { volùmene }\end{array}$




\section{Fontes documentarias}

CDS = Tola, Pasquale (ed.). 1858-1862. Codex Diplomaticus Sardiniae, 2 voll., Historiae Patriae Monumenta. Torino: Tip. Chirio e Mina.

CSG $=$ Meloni, Giuseppe (ed.). 2005. Il Condaghe di San Gavino. Cagliari: Cuec.

CSNT $=$ Merci, Paolo (ed.). 2001. Il Condaghe di San Nicola di Trullas. Nuoro: Ilisso.

Stat.Sass. = Guarnerio, Pier Enea (ed.). 1892. Gli Statuti della Repbblica Sassarese, testo logudorese del secolo XIV, nuovamente edito d'in sul codice. Archivio Glottologico Italiano XIII: 1-142.

\section{Bibliografia}

Aresu, Emilio. 2014a. Il ruolo della Sicilia alle origini del corso e del gallurese. Con una nota sul toponimo Palau. In Maxia, Mauro (ed.) Ciurrata di la Linga Gadduresa, Atti del I Convegno Internazionale di Studi, Palau 7 dicembre 2013. Olbia: Taphros, 165-170.

Aresu, Emilio. 2014b. Sardegna-Corsica-Sicilia. La lingua dei Galluresi sulla rotta delle grandi civiltà del Mediterraneo. Olbia: Taphros.

ATS 2006 = Loi, Antonio et alii. 2006. Atlante Toponomastico Sardo. Saggio metodologico. Regione Autonoma Sardegna/Università degli Studi di Cagliari/Università degli Studi di Sassari. Alghero: Poligrafica Peana.

ATS inèditu $=$ Atlante Toponomastico Sardo . Regione Autonoma Sardegna/ Università degli Studi di Cagliari/Università degli Studi di Sassari; rilevamenti effettuati dal 2007 al 2011.

Blasco Ferrer, Eduardo. 1984. Storia linguistica della Sardegna. Tubingen: De Gruyter.

Blasco Ferrer, Eduardo. 2010. Paleosardo: Le radici linguistiche della Sardegna neolitica. New York/Berlino: De Gruyter.

Brandanu, Salvatore. 2004. Vocabulàriu gaddhurésu italianu - Vocabolario gallurese-italiano. Olbia: Tipolitografia Sotgiu.

Casaccia, Giovanni. 1851. Vocabolario genovese-italiano. Genova: Fratelli Pagano.

Casu, Pietro. 2002. Vocabolario Sardo Logudorese - Italiano, ed. Giulio Paulis. Nuoro: ISRE.

Ciboddo, Pasquale. 2003. Dizionario Fondamentale Gallurese-Italiano. Sassari: Magnum-Edizioni.

De Felice, Emidio. 1964. Le coste della Sardegna. Saggio toponomastico storico-descrittivo. Cagliari: Fossataro. 
Ernout, Antoine \& Meillet, Alfred. 1951. Dictionnaire étymologique de la langue latine (3. a edizione). Parigi: Klincksiek.

Falcucci, Francesco Domenico. 1915. Vocabolario dei dialetti, geografia e costumi della Corsica, a cura di Pier Enea Guarnerio. Cagliari: Forni.

Gana, Leonardo. 1970. Vocabolario del dialetto e del folklore gallurese. Cagliari: Edizioni Della Torre.

Gismondi, Alfredo. 1955. Nuovo vocabolario genovese-italiano. Genova: Fides.

Malagoli, Giuseppe. 1939. Vocabolario pisano. Firenze: Lischi.

Maxia, Mauro. 1994. I nomi di luogo dell'Anglona e della Bassa Valle del Coghinas. Ozieri: Editrice Il Torchietto.

Maxia, Mauro. 2004. Il toponimo Billèllera e il blasone di Sorso. Rivista Italiana di Onomastica X/1. Roma.

Maxia, Mauro. 2011. Toponimi ricorrenti nel Mediterraneo occidentale. In Casanova, Emili et alii (ed.) La toponomàstica del les illes del Mediterrani occidental, XXXV Col·loqui de la Societat d'Onomàstica, L'Alguer maig 2008. Valencia-L'Alguer: Denes Editorial.

Maxia, Mauro. 2012. Fonetica storica del gallurese e delle altre varietà sardocorse. Olbia: Taphros.

Maxia, Mauro. 2015. L'elemento sardo del gallurese. In Atti della Seconda Giornata Internazionale della Lingua Gallurese, Palau 6 dicembre 2014. Olbia: Taphros (in cursu de editzione).

Naitana, Antonello. 2005. Le fonti del Monte Limbara. Tempio Pusania: Archimedia.

Panedda, Dionigi. 1991. I Nomi Geografici dell'Agro Olbiese. Sancasciano Val di Pesa (FI): Delfino.

Paulis, Giulio. 2006. Linee generali della Toponomastica Sarda. In Loi, Antonio et alii. Atlante Toponomastico Sardo. Saggio metodologico. Regione Autonoma Sardegna/Università degli Studi di Cagliari/Università degli Studi di Sassari. Alghero: Poligrafica Peana.

Paulis, Giulio. 1987. I nomi di luogo della Sardegna I. Roma: Delfino.

Piloni, Luigi. 1974. Carte geografiche della Sardegna. Cagliari: Edizioni Della Torre.

Pittau, Massimo. 1997. I nomi di paesi, città, regioni, monti, fumi della Sardegna. Significato e origine. Cagliari: Gasperini.

Pittau, Massimo. 2011. I toponimi della Sardegna. Significato e origine. Sassari: Edes.

Pittau, Massimo. 2014. Nuovo Vocabolario della Lingua Sarda etimologico e fraseologico I. Cagliari: Domus de Janas.

Puddu, Mario. 2000. Ditzionàriu de sa limba e de sa cultura sarda. Cagliari: Condaghes. 
Spanu, Pier Giorgio Ignazio. 2000. Martyria Sardiniae: i santuari dei martiri sardi. Oristano: Editrice S'Alvure.

REW = Meyer-Lübke, Wilhelm. [1935] 1968. Romanisches etymologisches Worterbuch. Heidelberg: Carl Winter.

Terracini, Benvenuto. 1926. Osservazioni sugli strati più antichi della toponomastica sarda. In Il Convegno Archeologico in Sardegna 1926. Sassari/ Roma, ristampa anastatica 1991, Delfino.

Tetti, Virgilio. 2001. I nomi di luogo, quarta dimensione della Sardegna II. Nuoro: Archivio Fotografico Sardo.

Toso, Fiorenzo. 2008. Linguistica di aree laterali ed estreme. Contatto, interferenza, colonie linguistiche e "isole" culturali nel Mediterraneo occidentale. Centro Internazionale sul Pluringuismo, Recco, Le Mani.

Usai, Andrea. 1977. Vocabolario tempiese-italiano italiano-tempiese. Sassari: Poddighe.

Wagner, Max Leopold. 1962. Dizionario Etimologico Sardo II. Heidelberg: Carl Winter.

Wagner, Max Leopold. 1984. Fonetica Storica del Sardo, Introduzione Traduzione e Appendice di Giulio Paulis. Cagliari: Trois. 